\title{
Development of a System for Evaluating Abutment Teeth
}

\author{
Takeshi Murayama,, \# Mana Kawakami, Kozue Miyamoto," Toru Eguchi, \\ Mitsuhiro TAмамото*
}

\begin{abstract}
In dental treatment, dental computer-aided design (CAD) and computer-aided manufacturing (CAM) systems have been introduced to assist dental technicians in fabricating dental prostheses. The fabrication process is as follows. Before fabrication, the dentist removes dental caries from a patient's tooth and prepares an abutment tooth. Next the shape of the abutment tooth is scanned by a 3-D scanner to generate a 3-D model of the abutment tooth on a computer. Then, using a CAD system, the dental technician designs a dental prosthesis that fits the abutment tooth. Finally, the dental prosthesis is fabricated using a CAM system and a machine tool. In the process of using the CAD/CAM systems, the shapes of abutment teeth sometimes cause problems such as the impossibility of fabricating a dental prosthesis. The purpose of this study was to develop a system that evaluates the appropriateness for the abutment teeth. Using 3-D models of abutment teeth, the system can examine: ( 1 ) whether a prosthesis can be fabricated using the machine tool; and ( 2 ) whether there are undercuts on the abutment tooth. Using the system, we evaluated the abutment teeth prepared by students in our dental training school. The evaluation by the system was compared with the assessment by a teacher. The system execution and the comparison showed that the system can be used for evaluating abutment teeth.
\end{abstract}

Keywords : dentistry, abutment teeth, evaluation, dental computer-aided design, computer-aided manufacturing, 3-D models.

Adv Biomed Eng. 1: pp. 23-26, 2012.

\section{Introduction}

In dental treatment, dental computer-aided design (CAD) and computer-aided manufacturing (CAM) systems have been introduced to assist dental technicians in fabricating dental prostheses. The fabrication process is as follows:

(1) Before fabrication, the dentist removes dental caries from a patient's tooth and prepares an abutment tooth, as shown in Fig. 1 (a).

( 2 ) The shape of the abutment tooth is scanned by a 3 -D scanner to generate a 3-D model of the abutment tooth on a computer;

( 3 ) Using a CAD system, the dental technician designs a dental prosthesis that fits the abutment tooth, as shown in Fig. 1 ( b ).

(4) Using a CAM system and a machine tool, the dental prosthesis is fabricated by cutting the material, as shown in Fig. 1 ( $\mathbf{c}$ ).

In the process of using the $\mathrm{CAD} / \mathrm{CAM}$ systems, the shapes of abutment teeth sometimes cause problems such

This study was presented at the Symposium on Biomedical Engineering 2012, Suita, September, 2012.

Received on July 27, 2012; revised on September 19, 2012 and October 10, 2012; accepted on October 10, 2012.

* Graduate School of Biomedical \& Health Sciences, Hiroshima University, Hiroshima, Japan.

** Graduate School of Engineering, Hiroshima University, Hiroshima, Japan.

\# 1-2-3 Kasumi, Minami-ku, Hiroshima 734-8553, Japan. E-mail:murayatk@hiroshima-u.ac.jp
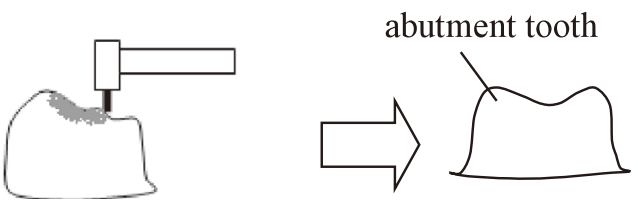

(a) Removal of dental caries and preparation of an abutment tooth

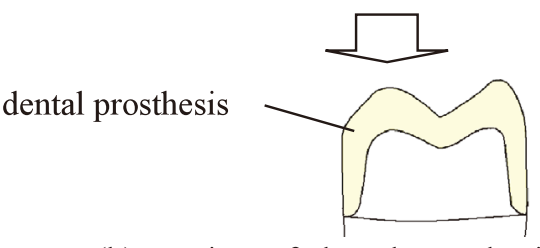

(b) Design of dental prosthesis to fit the abutment tooth

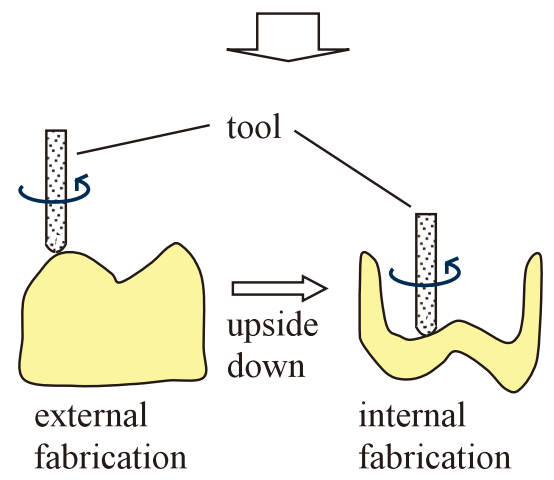

(c) Fabrication of dental prosthesis by cutting the material

Fig. 1 Fabrication process of dental prosthesis. 
as impossibility of fabricating a dental prosthesis. The purpose of this study was to develop a system that evaluates the appropriateness of the abutment teeth.

Other preparation assistant systems (e.g., Cadim, PREPassist, ClinSim) have been developed [1, 2] for the purpose of dental preparation training in dental training schools. These systems identify the difference between the 3-D model of an abutment tooth prepared by a student and that of an ideal abutment tooth sometimes prepared by a teacher. These systems require ideal models for evaluation of abutment teeth, but it is impossible to have such models in actual patient treatment. In contrast, our system does not require the ideal models of abutment teeth since our system analyzes the anatomic features of abutment teeth to evaluate their appropriateness.

\section{Required Conditions for Abutment Teeth}

There are many required conditions for abutment teeth in order not to cause problems in prosthesis fabrication [3]. In the present study, we focus on the following two conditions.

( 1 ) The abutment teeth should not have any corners that are sharper than the edge of the tool. If an abutment tooth has such corner, the inside surface of the dental prosthesis cannot be fabricated by the machine tool, as shown in Fig. 2.

(2) The abutment teeth should not have any undercut. As shown in Fig. 3, such undercuts on the abutment teeth make the insertion of the dental prosth-

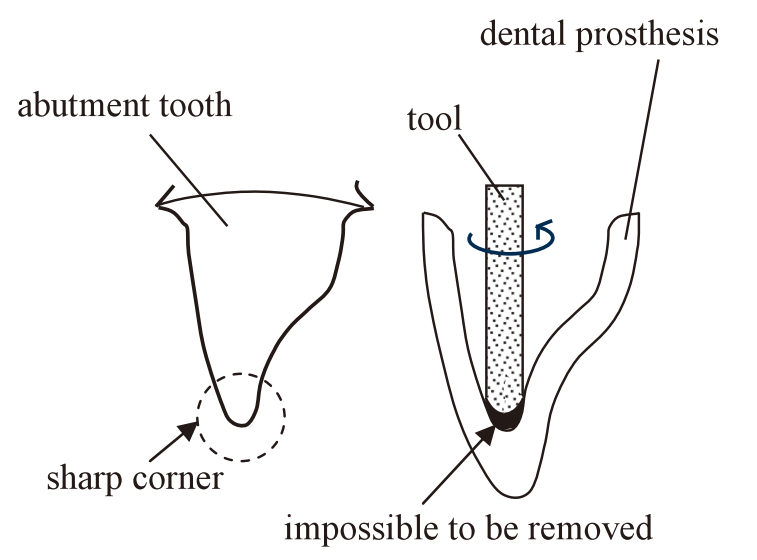

Fig. 2 Impossible to fabricate prothesis because of a sharp corner.

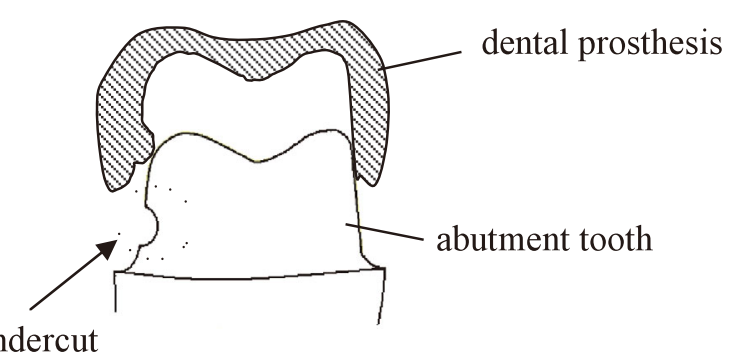

Fig. 3 Impossible to insert because of an undercut. eses to the patients' abutment teeth impossible. In addition, the undercuts render scanning of the abutment teeth difficult or sometimes impossible.

\section{Method of Checking whether Abutment Teeth Satisfy the Required Conditions}

We developed a system to check whether the abutment teeth satisfy the required conditions mentioned above. The system inputs the 3-D model of an abutment tooth. The 3-D model we use is a polygon model composed of numerous small polygons, as shown in Fig. 4. Each polygon is described by the positions of three vertexes and a normal vector.

\subsection{Examining the feasibility of fabricating dental prostheses}

Using the 3-D model, the system examines whether a

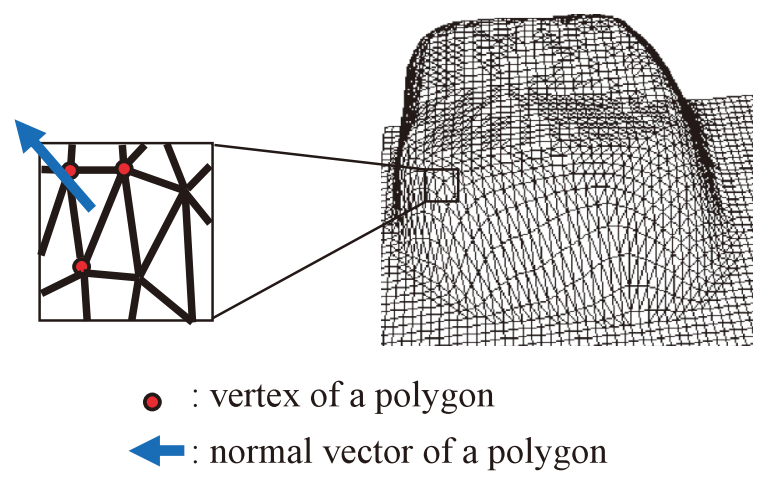

Fig. 4 3-D model of an abutment tooth.

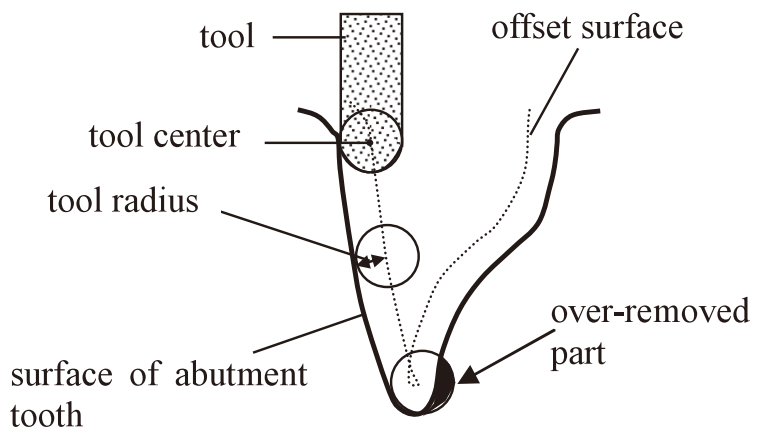

(a)

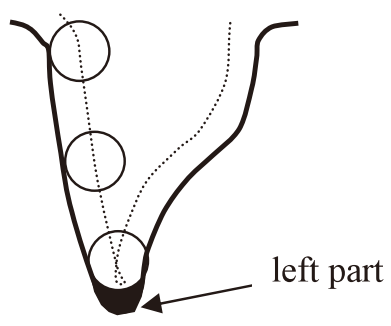

(b)

Fig. 5 Rolling of a virtual ball to check the possibility of fabrication. 
prosthesis can be fabricated by the following procedures. First, the system generates the offset surface at a specific distance, which equals to the tool radius, from the original surface of the abutment tooth. The surface of the abutment tooth is identical to the inside surface of the dental prosthesis to be made, and the offset surface represents the surface that the tool center passes along. In Fig. 5, the dotted line shows the offset surface. Next, the system moves a virtual ball that mimics the tool and has a radius identical to the tool radius. As the center of the ball passes along the offset surface, if the ball protrudes from the surface of the abutment tooth, the protruding part will be over-removed compared to the original surface, as shown in Fig. 5 ( a ). Conversely, to avoid over-removal, a part that requires removed cannot be removed and is left, as shown in Fig. 5 (b). Therefore, if the ball protrudes from the surface of the abutment tooth, the system judges that the prosthesis cannot be fabricated properly.

\subsection{Examining whether there are undercuts on abutment teeth}

The system searches the 3-D model of an abutment tooth for polygons with normal vectors directing downward. The downward polygons represent area of undercut, as shown in Fig. 6. However, as shown in Fig. 7, the downward polygons are depicted differently (i.e., the area of undercut is different), depending on the pose of the abutment tooth. Therefore the system searches for a pose, in which the number of downward polygons is zero, using a genetic algorithm which is a search method mimicking

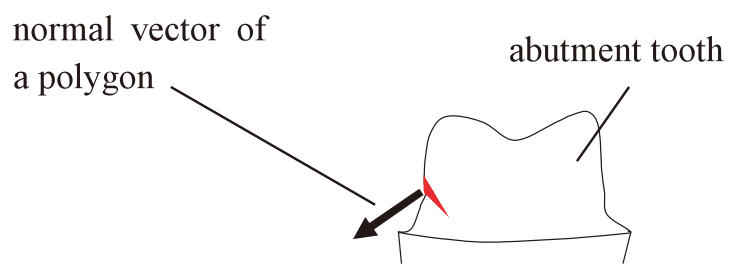

Fig. 6 Downward normal vector of a polygon in a 3-D model of abutment tooth. The red part shows the area of undercut.
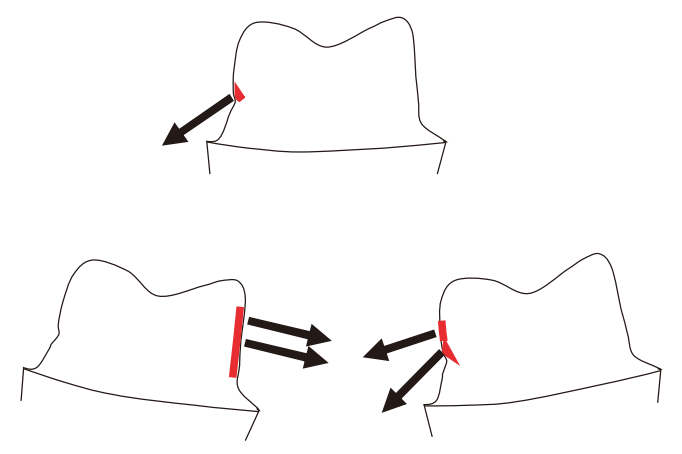

Fig. 7 Different downward polygons depending on the pose of the abutment tooth. The red parts show the areas of undercuts. the process of natural evolution. If the system finds a pose in which there are no downward polygons, the system judges that the abutment tooth has no undercuts.

The procedure of the genetic algorithm, which is utilized to search for poses, is as follows.

Step 1: Start with a set of randomly generated poses of the abutment tooth. The set is called "population”. Each pose is represented by two angles $\left(\theta_{x}, \theta_{y}\right)$. These angles express rotations around $\mathrm{x}$ and $\mathrm{y}$ axes, respectively, from an initial pose to the pose tested. We do not have to take account of the rotation around $z$ axis because the rotation around $z$ axis does not change the area of the undercut. Each of the angles around $\mathrm{x}$ and $\mathrm{y}$ axes is represented by $\mathrm{a}$ binary string. For example, an angle of 5 degrees is represented by 000101 as the binary number. Therefore each of the poses is represented by two binary strings corresponding to $\left(\theta_{x}, \theta_{y}\right)$.

Step 2: If the population includes a pose in which there are no downward polygons, stop and judge that the abutment tooth has no undercuts. If the number of repetition from Step 2 to Step 7 reaches a predetermined number and the population does not include any pose with no downward polygons, stop and judge that the abutment tooth has undercut. Otherwise, go to the next step.

Step 3: Calculate the fitness value, $f_{i}$, for each pose, $i$, by:

$$
f_{i}=\frac{1}{D_{i}}
$$

where $D_{i}$ is the number of downward polygons in pose $i$. Therefore, larger $f_{i}$ is associated with smaller area of undercut.

Step 4: Select a pair of poses from the current population, the probability of selection being an increasing function of fitness value. Therefore the poses with large fitness values (i.e., small areas of undercut) have high possibilities of being selected. We call the selected poses "parent poses."

Step 5: Cross over the pair using a randomly made mask to create two new poses. In the example shown in Fig. 8, at the loci showing 1 in the mask, the new pose $C$ inherits from the parent pose $A$ and the new pose $D$ inherits from the parent pose $B$. In contrast, at the loci showing 0 in the mask, the new pose $C$ inherits from the parent pose $B$ and the new pose $D$ inherits from the parent pose $A$.

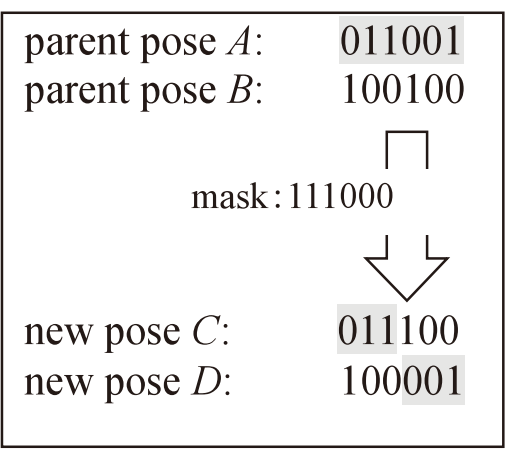

Fig. 8 An example of crossover. 


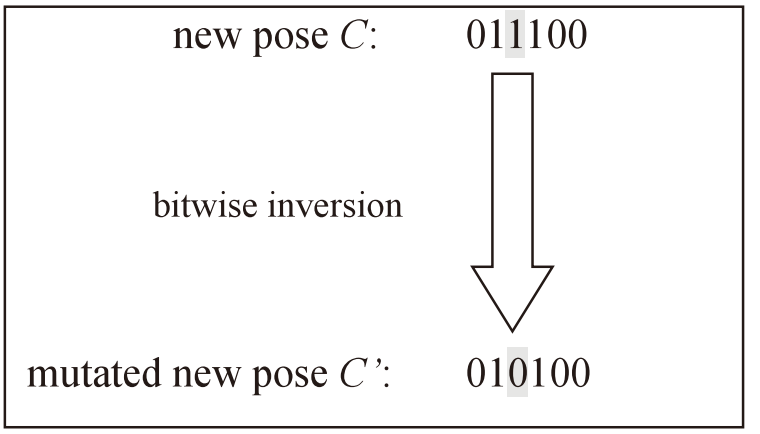

Fig. 9 An example of mutation.

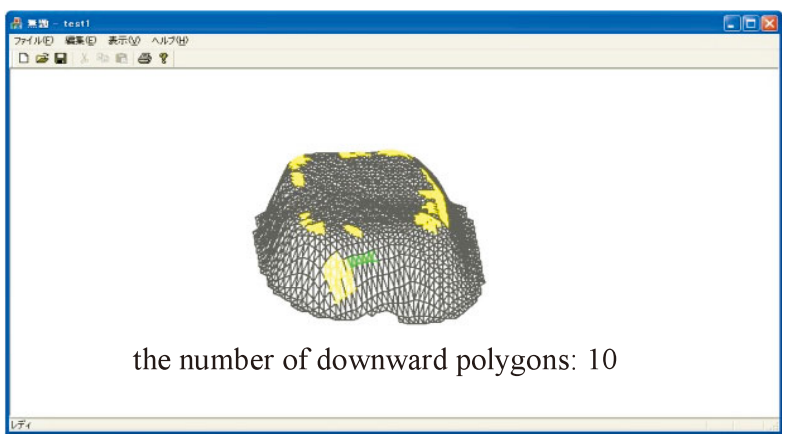

Fig. 10 Result of evaluation performed by the system.

Step 6: Mutate the two new poses at each locus with the given mutation probability. The mutation is the bitwise inversion at a randomly selected locus in the new poses, as shown in Fig. 9.

Step 7: Replace the current population with the new population including the new poses generated through crossover and mutation, and go to Step 2 .

\section{System Execution}

Using the system, we evaluated the abutment teeth prepared by students in our dental training school. Figure 10 shows an example of the result of evaluation. In this figure, the green part shows the area of undercut and the yellow part shows the part causing impossibility of fabricating the prosthesis. The evaluation by the system was compared with the assessment by a teacher. The judgments of both the possibility of fabrication and existence of undercuts by the system were the same as those of the teacher. The teacher commented that the system can be used for evaluation of abutment teeth prepared by students.

\section{Conclusions}

We developed a system that evaluates the appropriateness of abutment teeth from the viewpoints of feasibility of fabrication and presence of undercuts. The system execution and comparison showed that the system can be used for evaluating abutment teeth. We are now expanding the system to examine whether the abutment teeth satisfy other required conditions.

\section{References}

1. Jaeger B, Kournetas N, Groten M, Lachmann S, Weber H, Geis-Gerstorfer J:PREPassist as digital assistance for dental education. Int Poster J Dent Oral Med. 5, p. 169, 2003.

2. Kournetas N, Jaeger B, Axmann D, Groten M, Lachmann $\mathrm{S}$, Weber H, Geis-Gerstorfer J: Assessing the reliability of a digital preparation assistant system used in dental education. J Dent Educ. 68 (12) , pp. 1228-1234, 2004.

3. Sadan A, Blatz MB, Lang B:Clinical considerations for densely sintered almina and zirconia restorations: Part 1 . Int J Periodontics Restorative Dent. 25 (3), pp. 213-222, 2005.

\section{Takeshi Murayama}

Takeshi Murayama is a professor in Graduate School of Biomedical and Health Sciences at Hiroshima University since 2005. He received his master degree from University of Osaka Prefecture in 1986 and his doctor degree from Hiroshima University in 1993.

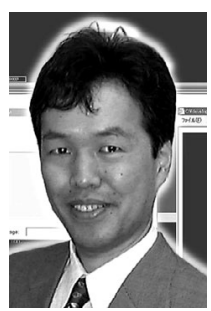
His current interests include medical systems and the application of manufacturing system technologies (e.g., $\mathrm{CAD} / \mathrm{CAM}$, rapid prototyping, concurrent engineering, production planning and control) to dentistry. 